\title{
Indirect CLEM Identifies Proarrhythmic Remodeling of Intercalated Disk Nanodomains in Murine Atria Following Acute VEGF Treatment
}

\author{
Louisa Mezache ${ }^{1}$, Heather Struckman ${ }^{1}$, Amara Greer-Short ${ }^{2}$, Thomas J Hund ${ }^{1,2}$, Rengasayee \\ Veeraraghavan ${ }^{1,2 *}$ \\ 1. Department of Biomedical Engineering, College of Engineering, The Ohio State University, \\ Columbus, OH, USA. \\ 2. Bob and Corinne Frick Center for Heart Failure and Arrhythmia Research, The Ohio State University, \\ Columbus, OH, USA. \\ * Corresponding author: Veeraraghavan.12@osu.edu
}

Previous work by us [1,2], and others [3,4], suggests that nanodomains, enriched with the cardiac sodium channel ( $\mathrm{Na} v 1.5)$, within the intercalated disk play a key role in cardiac impulse propagation. Further, we recently demonstrated deadhesion within these ID nanodomains in patients with atrial fibrillation (AF) [5]. In experimental studies, such nanodomain deadhesion was prompted by acute edema $[2,6]$. We therefore hypothesized that elevated levels of vascular leak-inducing cytokines, such as VEGF [7-9], in AF patients may contribute to arrhythmia by disrupting ID nanodomains.

In order to test this hypothesis, ex vivo murine hearts were treated with a clinically relevant VEGF dose (500 ng/l) and ID structure and molecular organization were evaluated using indirect correlative light electron microscopy (indirect CLEM). Briefly, indirect CLEM utilizes structural fiducials such as the edges of gap junctions (GJ) and mechanical junctions (MJ) to correlate ultrastructure, assessed by transmission electron microscopy (TEM), with molecular organization, assessed by STochastic Optical Reconstruction Microscopy (STORM). Thus, separate tissue samples from each heart were fixed for TEM, and cryopreserved for STORM. The results from the two methods are then correlated using structural fiducials - specifically the edges of GJ and MJ, identifiable by TEM, correlate to the edges of connexin43 (Cx43) and N-cadherin (N-cad) clusters, identifiable by STORM. Thus, indirect CLEM affords the advantages of correlating ultrastructure with molecular organization, while retaining the high throughput of TEM and STORM.

TEM revealed close apposition of adjacent cells near GJ $(17 \pm 1 \mathrm{~nm})$ and MJ (27 $\pm 2 \mathrm{~nm})$. Machine learning-based STORM-RLA[10] analysis revealed preferential enrichment of Nav1.5 near Cx43 at a median distance of $70 \pm 4 \mathrm{~nm}$. Cx43-adjacent sites $(<200 \mathrm{~nm})$ accounted for $45 \pm 2 \%$ of ID-localized $\mathrm{Na}_{\mathrm{V}} 1.5$, which was organized into dense clusters $(9 \pm 2$ folder higher density compared to non-junctional ID sites). N-cad-rich sites (MJs) accounted for an additional $39 \pm 1 \%$ of ID-localized Nav1.5 with $7 \pm 1$ fold higher density than non-junctional ID sites.

Following 60 minutes of VEGF treatment, intermembrane spacing, assessed by TEM, was increased adjacent to GJ (64 $\pm 9 \mathrm{~nm}, \mathrm{p}<0.05$ vs. control) and $\mathrm{MJ}(63 \pm 4 \mathrm{~nm}, \mathrm{p}<0.05$ vs. control). These data are consistent with deadhesion within ID nanodomains secondary to VEGF-mediated vascular leak. STORM-RLA detected no change in inter-cluster distance from GJ and MJ to Nav1.5. However, VEGF treatment significantly reduced Nav1.5 cluster density near GJs ( $6 \pm 1$ fold over non-junctional ID sites) and MJs ( $4 \pm 1$ fold over non-junctional ID sites) compared to control. These data are consistent with Nav1.5 translocation from Cx43-adjacent sites following VEGF treatment.

Finally, we examined the functional impacts of VEGF-induced ID nanodomain remodeling. VEGF 
slowed atrial conduction as assessed by optical voltage mapping ( $10 \pm 0.4 \mathrm{vs} .21 \pm 1 \mathrm{~cm} / \mathrm{s}$ at baseline, $p$ $<0.05)$. Burst pacing induced AF in 4 hearts out of 5 tested following VEGF treatment but not before.

In summary, indirect CLEM identifies ID nanodomain deadhesion and Nav1.5 translocation following acute VEGF treatment. Importantly, these structural changes correlated with slowed atrial conduction and increased AF inducibility. These results suggest that vascular leak-induced ID nanodomain remodeling may contribute to the development of AF.

\section{References:}

[1] R Veeraraghavan et al., Elife 7 (2018), e37610.

[2] R Veeraraghavan et al., Pflugers Arch 467 (2015), p. 2093.

[3] A Leo-Macias et al., Nat Commun 7 (2016), p. 10342.

[4] A Leo-Macias, FX Liang and M Delmar, Cardiovascular research 107 (2015), p. 442.

[5] TB Raisch et al., Front Physiol, 9 (2018), p. 398.

[6] R Veeraraghavan, ME Salama and S Poelzing, American Journal of Physiology Heart and Circulatory Physiology 302 (2012), p. H278.

[7] NA Chung et al., Stroke 33 (2002), p. 2187.

[8] J Li et al., Heart rhythm : The Official Journal of the Heart Rhythm Society 7 (2010), p. 438.

[9] Y Seko et al., Jpn Heart J 41 (2000), p. 27.

[10] R Veeraraghavan and R Gourdie, Molecular biology of the cell 27 (2016), p. 3583.
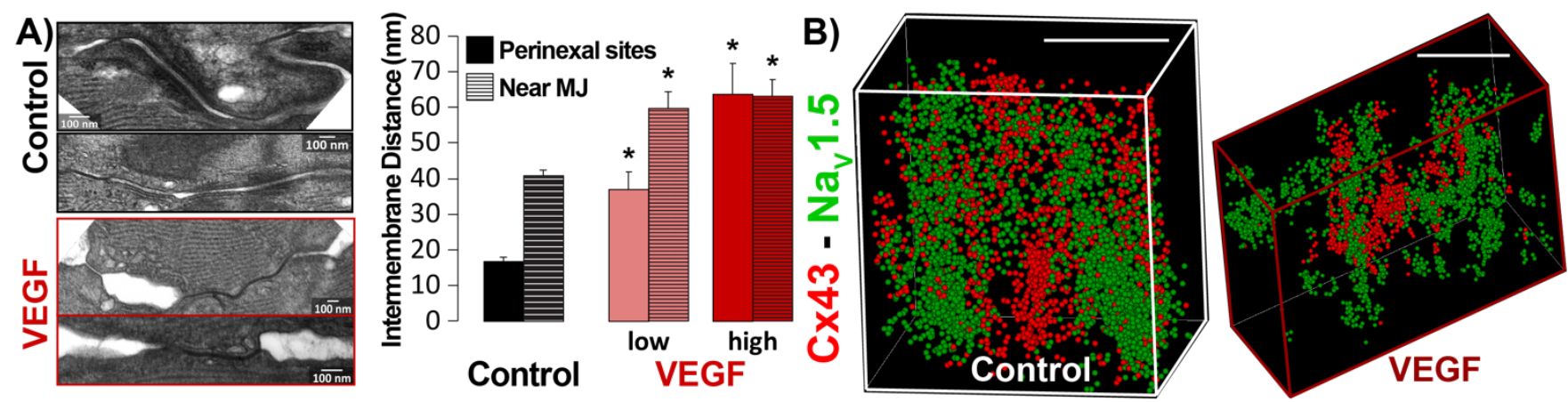

Figure 1. A) TEM demonstrates de-adhesion near GJ and MJ following 60 minutes of VEGF at clinically relevant doses (low: $100 \mathrm{pg} / \mathrm{ml}$, high: $500 \mathrm{ng} / \mathrm{l}$ ). B) Close-up 3D STORM images illustrate Nav1.5 enrichment adjacent to $\mathrm{Cx} 43$ GJs in control mouse atria (left) and Nav1.5 translocation following VEGF-treatment (right). Scale bars: $1 \mu \mathrm{m}$. 